\title{
A novel angiogenesis model for screening anti-angiogenic compounds: The chorioallantoic membrane/feather bud assay
}

\author{
HAIMING CHEN ${ }^{1}$, CATHY S. WANG ${ }^{1}$, MINGJIE LI ${ }^{1}$, ERIC SANCHEZ ${ }^{1}$, JENNIFER LI ${ }^{1}$, \\ ARIANA BERENSON $^{1}$, ERIC WIRTSCHAFTER ${ }^{1}$, JAMES WANG ${ }^{1}$, JING SHEN ${ }^{1}$, \\ ZHIWEI LI $^{1}$, BENJAMIN BONAVIDA ${ }^{2}$ and JAMES R. BERENSON ${ }^{1}$
}

\author{
${ }^{1}$ Institute for Myeloma \& Bone Cancer Research, West Hollywood; ${ }^{2}$ Departments of Microbiology, Immunology and \\ Molecular Genetics, Geffen School of Medicine at the University of California at Los Angeles, Los Angeles, CA, USA
}

Received March 23, 2010; Accepted April 19, 2010

DOI: 10.3892/ijo_00000654

\begin{abstract}
Enhanced angiogenesis is a hallmark of solid tumors and hematological malignancies. Anti-angiogenic therapeutic approaches have recently been shown to be effective for the treatment of certain cancers. Endothelial cells migrating to tumors provide them with new blood vessels that are critical for their growth and survival. We have developed a novel and rapid method to evaluate the anti-angiogenic activity of new agents consisting of a combined chorioallantoic membrane (CAM) and feather bud (FB) assay. Unlike previous assays, this new assay assesses the effects of drugs on the ability of tissues to attract and develop their own blood supply. The CAM already has a well-developed vascular network that is capable of providing blood vessels to the non-vascularized $\mathrm{FB}$, allowing for this tissue to develop feathers. As a result, the exposure of the FB to drugs for 2 days followed by attachment to the CAM for 4 days allows evaluation of the compound's ability to impact blood vessel and feather formation within the CAM-attached FB tissue. Feather formation is determined as well as expression of endothelial cell genes and proteins analyzed. Using agents with known anti-angiogenic activity including fumagillin, minocycline, zoledronic acid, doxorubicin and agents lacking anti-angiogenic activity such as melphalan, we have shown that the CAM/FB assay can accurately and rapidly assess the ability of agents to prevent blood vessel and feather development within non-vascularized tissues.
\end{abstract}

Correspondence to: Dr James R. Berenson, Institute for Myeloma \& Bone Cancer Research, 9201 West Sunset Blvd., Suite 300, West Hollywood, CA 90069, USA

E-mail: jberenson@imbcr.org hchen@imbcr.org

Abbreviations: CAM, chorioallantoic membrane; FB, feather bud

Key words: angiogenesis, chorioallantoic membrane, feather bud assay

\section{Introduction}

Angiogenesis is a complex process involving endothelial cell proliferation and migration leading to the development of blood vessels from pre-existing vasculature and involves sequential events including proteolysis and remodeling of the extracellular matrix (1). Furthermore, angiogenesis and vasculogenesis, the development of new blood vessels, play an important role in the induction and maintenance of a variety of pathological states. Some of these manifestations are characterized by neovascularization, including diabetic retinopathy, glaucoma, age-related macular degeneration and cancer (2-4). Angiogenesis is essential for the growth and metastasis of malignant tumors. Endothelial cells recruited to tumors enrich them with new blood vessels that are critical for their growth and survival, and anti-angiogenic therapy represents one of the most promising new approaches for cancer treatment $(5,6)$. Anti-angiogenic therapeutic approaches have recently shown clinical benefit in a variety of cancers $(7,8)$. Our recent identification of pleiotrophin as a major factor in the early development of tumor blood vessels through transdifferentiation of monocytes into vascular endothelium provides an opportunity to develop new agents to block this pro-vasculogenic effect (9). There remains an urgent need to develop strategies to more rapidly, inexpensively and accurately assess the pro-angiogenic and anti-angiogenic activities of new biologic factors and putative therapeutic agents.

Both in vitro and in vivo methods have been previously established to identify compounds which either stimulate or inhibit angiogenesis and/or vasculogenesis. In vitro assays include the Matrigel ${ }^{\mathrm{TM}}$ tube-forming assay $(10,11)$, fibrin and collagen gel-cord-forming assays $(12,13)$, the aortic ring model (14) and a variety of endothelial cell proliferation assays (10). The drawback of these assays is that they are incapable of assessing the important interactions that take place between multiple cell types involved in the development of blood vessels and specifically the ability of tissues to attract their own blood supply. Recently, in vivo assays have been developed to analyze angiogenesis and/or vasculogenesis in a more clinically relevant manner. These methods include the rat, mouse and rabbit corneal pocket $(15,16)$, primate iris 
neovascularization (17), human/mouse chimeric angiogenesis (18) and murine Matrigel plug assays (19). However, these assays are limited in their applicability by the prolonged length of time they require to assess anti-angiogenic effects, their complexity, inclusion of non-viable material such as Matrigel, artificial addition of cytokines or other factors, animal-related morbidity and high costs required to carry them out. The CAM assay with polyester sponges or Matrigel has been developed as an angiogenesis assay (20-22). The CAM develops from the fusing of the mesodermal layer of the allantoic membrane with the same layer of the chorion which completely surrounds the embryo during embryonic development. It is attached to the internal surface of the shell membrane as well as providing a barrier between the watery environment of the embryo and the air space. However, this method involves the placement of Matrigel or other artificial material such as sponges at the site for testing the potential anti-angiogenic effects of compounds. In addition, these methods have often included the addition of exogenous factors to artificially stimulate angiogenesis. Importantly, all of these previously developed assays evaluate the direct impact of agents on already vascularized tissues and are unable to assess the effect of drugs on a tissue's ability to attract blood vessels, what we term so-called angioattraction, and their further development within those tissues. We have developed a novel method to assess the effects of agents on angioattraction and the further development of these blood vessels within tissues lacking vascularity in a rapid, accurate and inexpensive way without the inclusion of artificial testing materials or exogenous factors.

\section{Materials and methods}

Drugs. Zoledronic acid was supplied by Novartis Pharmaceuticals (Basel, Switzerland). Melphalan, minocycline and fumagillin were purchased from Sigma (St. Louis, MO), and doxorubicin was purchased from Calbiochem (La Jolla, CA).

Preparation of the FB. Fertilized chick eggs (Charles River, Wilmington, MA) were incubated horizontally at $37.5^{\circ} \mathrm{C}$ in a humidified incubator for 8 days and staged according to Hamburger and Hamilton (1951). Stage 33 chick embryonic dorsal skin with FBs was collected under a dissecting microscope (Olympus) in Hank's buffered saline solution (Gibco/ Invitrogen). The FB skin was cut into $2 \times 2 \mathrm{~mm}$ sections and placed on culture inserts in 6-well culture dishes (Falcon). The FBs were cultured with DMEM containing $2 \%$ fetal calf serum, gentamicin $(1: 1000)$ and with or without drugs at $37^{\circ} \mathrm{C}$ with an atmosphere of $5 \%$ carbon dioxide air for $48 \mathrm{~h}$. Images of the FBs were analyzed by dissection microscopy to determine size, area, shape factor and orientation of each FB.

FB/CAM co-culture. A further set of fertilized chick eggs was incubated horizontally at $37.5^{\circ} \mathrm{C}$ in a humidified incubator and windowed by day 8 . The $2 \times 2 \mathrm{~mm}$ FBs treated with drugs or without drugs was transferred onto the CAM of an 8-day-old chick embryo with sterile fine forceps from the culture insert. For this step, room conditions included a temperature of $70-75^{\circ} \mathrm{C}$ with $40-50 \%$ humidity. The eggs were sealed with an adhesive tape and incubated for an additional 2-4 days.
The endothelial cells of CAM proliferated and migrated into the FB after 2 days. After 4 days of culture, both blood vessel formation and FB development were determined by microscopy. Photographs were taken at described stages using transillumination with an Olympus IMT 2 inverted microscope. To measure the FB development, the feather weight of each stage was measured by fine balance. At least three independent experiments were carried out for each drug and each showed similar results.

Immunohistochemical (IHC) and immunofluorescent analysis (IFA) of vascular endothelial cell markers in feather development. Chicken embryonic skin samples of FBs cultured on CAM were fixed with $4 \%$ paraformaldehyde in PBS and dehydrated in a graded series of ethanol. The samples were processed with a standard paraffin-embedding procedure and dissected into five micron sections. After removing the wax by a standard paraffin heating process, the slides were blocked with $0.05 \%$ Tween-20 (TBST) and 3\% BSA for $1 \mathrm{~h}$ at room temperature. The slides were exposed to anti-Tie-2 antibodies (Santa Cruz Biotech, Santa Cruz, CA) overnight. They were then washed three times with TBST and treated with $\mathrm{H}_{2} \mathrm{O}_{2}$ for $3 \mathrm{~min}$ to remove endogenous peroxidase. Horseradish peroxidase (HRP)-conjugated with anti-mouse IgG (KPL, Gaithersburg, MD) was diluted 1:500 in TBST and incubated with slides at RT for $2 \mathrm{~h}$. The slides were then washed three times in TBST, placed in a 3-amino-9ethylcarbazole (AEC) buffer for $5 \mathrm{~min}$ and color was detected using an AEC kit (Vector). Endothelial markers were identified under the BX51 (Olympus) microscope. For IFA, the slides were blocked with $0.05 \%$ Tween-20 (TBST) and 3\% BSA for $1 \mathrm{~h}$ at RT and were incubated with anti-mouse $\mathrm{IgG}$ conjugated to phycoerythrin (PE) (1:100; BD Biosciences, San Jose, CA) at $4^{\circ} \mathrm{C}$ overnight. The slides were washed three times with PBS for $15 \mathrm{~min}$ at RT and incubated with FITC-conjugated swine anti-goat or anti-mouse antibody (Biosource, Camarillo, CA) for $2 \mathrm{~h}$ at RT. Anti-DAPI antibody was added to slides as a nuclear marker. The slides were washed as previously mentioned and mounted with aqueous mounting media (Biomeda, Foster City, CA). Endothelial markers were identified under the microscope, and merged cells were analyzed using the Microsuite Biological Suite program (Olympus BX51, San Diego, CA).

Western blot analysis. Protein lysates $(20 \mu \mathrm{g})$ from the FB were electrophoresed on a 4-15\% SDS-polyacrylamide gel, $100 \mathrm{~V}$ for $3 \mathrm{~h}$ at $4^{\circ} \mathrm{C}$ and then proteins were transferred to polyvinylidene difluoride membranes (Bio-Rad, Hercules, CA) overnight at $50 \mathrm{~mA}, 4^{\circ} \mathrm{C}$. The membranes were incubated with 5\% BSA in TBST for $1 \mathrm{~h}$ at RT. Anti-Tie-2, anti-Flk-1 or anti-GAPDH antibodies were added and incubated overnight at $4^{\circ} \mathrm{C}$. Protein expression was visualized using an enhanced chemiluminescence detection system (Amersham, Buckinghamshire, UK) and quantified using a Vesa-Doc gel documentation system (Bio-Rad).

$R T-P C R$. Total RNA was isolated from each feather treated with or without fumagillin, minocycline or zoledronic acid at different concentrations. RNA was re-suspended in $0.1 \%$ diethyl pyrocarbonate-treated water, digested with DNase I 

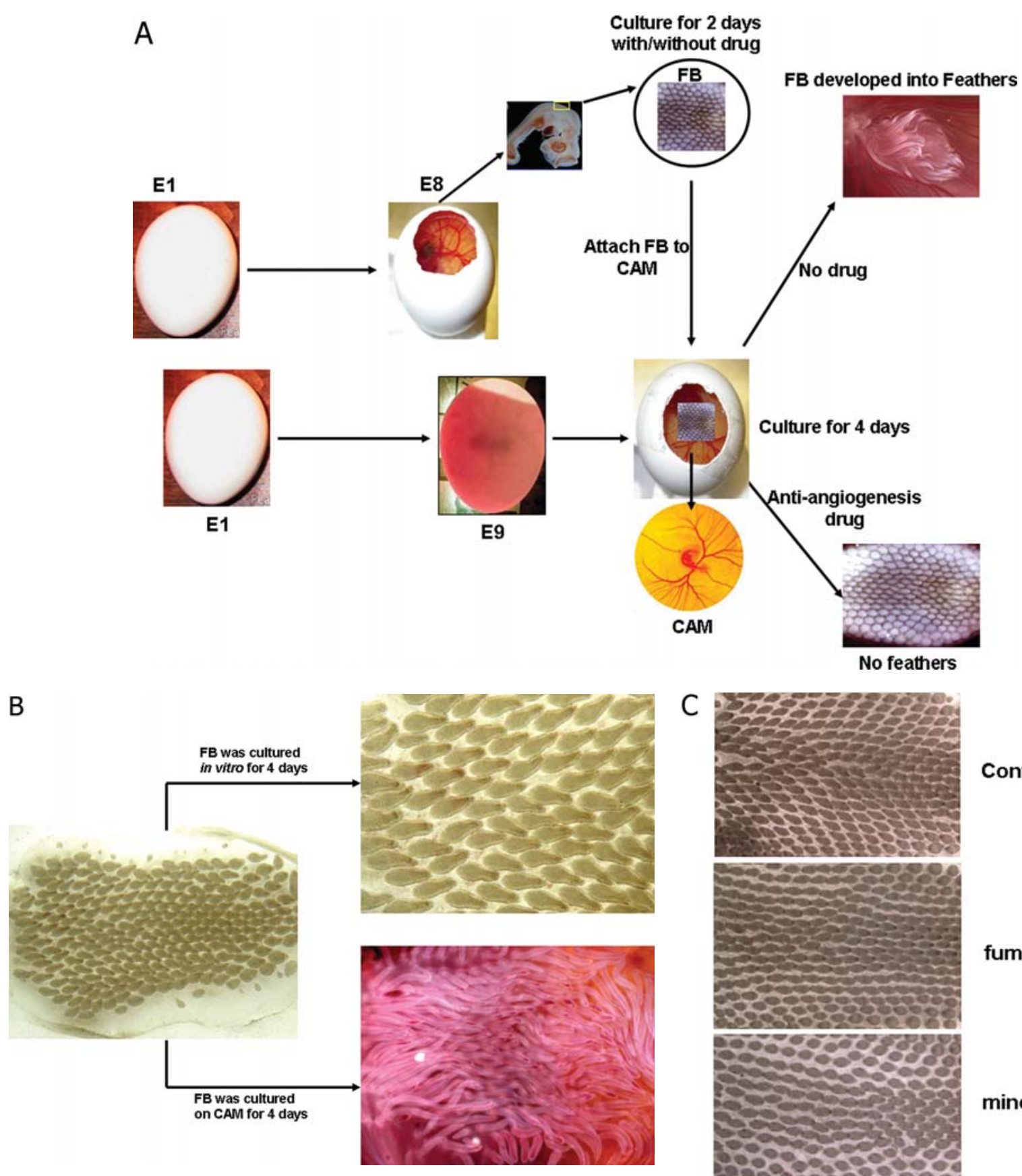

C

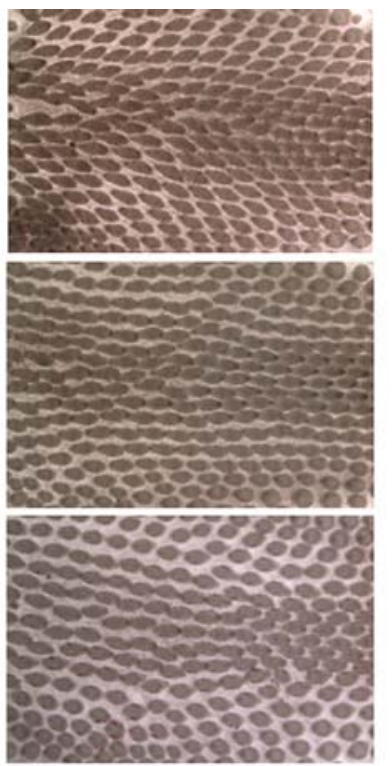

Control

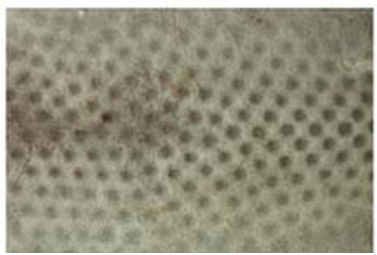

fumagillin $(1 \mu \mathrm{M})$

minocycline $(5 \mu \mathrm{M})$

melphalan $(30 \mu \mathrm{M})$

Figure 1. (A) Fertilized chick eggs were placed in an incubator and kept under constant humidity at $37^{\circ} \mathrm{C}$. On day 8 , a window was opened in the shell and the embryo placed in a Petri dish. The embryonic skin was detached from the body and cut into $5 \mathrm{~mm}^{2}$ pieces. The FB was cultured in culture medium with or without anti-angiogenesis reagents for $48 \mathrm{~h}$. The treated FB was loaded onto E9 CAM and the window sealed. Angiogenesis and feather bud development were examined after 4 days of culture. The feather formation was markedly inhibited by anti-angiogenesis drugs. (B) Development of FB: left, the size of the FB derived from E8 chicken embryonic skin increased 5-fold after culturing the FB in vitro for 4 days. Right, blood vessels migrated into the FB and endothelial cells proliferated in the feather by culturing the FB on CAM for 4 days. (C) In vitro culture of the FB, embryonic skins were collected from E8 chick eggs under a dissecting microscope to isolate the FB. The FB was exposed to drugs for two days by incubating the FB in culture medium with either anti-angiogenesis compounds ( $1 \mu \mathrm{M}$ fumagillin or $5 \mu \mathrm{M}$ minocycline) or a chemotherapeutic drug without anti-angiogenic effects (30 $\mu \mathrm{M}$ melphalan) for $48 \mathrm{~h}$. Whether the FB diminished or disappeared with exposure to drugs was determined using dissecting microscopy.

(Sigma) to remove contaminating DNA and extracted with phenol/chloroform followed by ethanol precipitation. Total
RNA $(1 \mu \mathrm{g})$ was reverse-transcribed to cDNA and amplified using the ThermoScript RT-PCR System (Invitrogen, Carlsbad, 


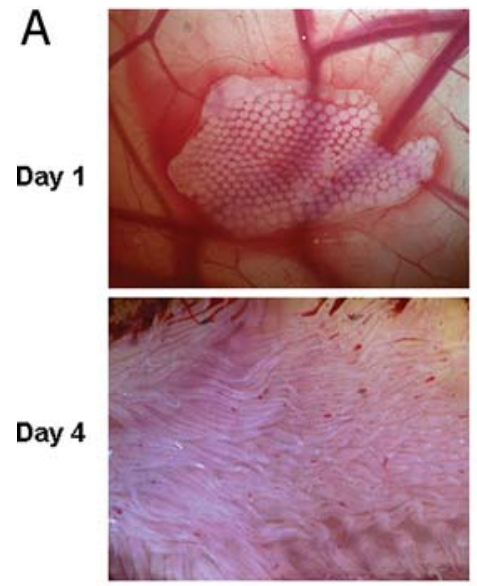

Control (PBS)

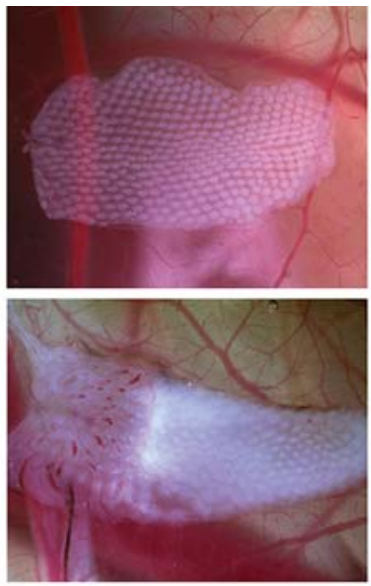

$(0.1 \mu \mathrm{M})$
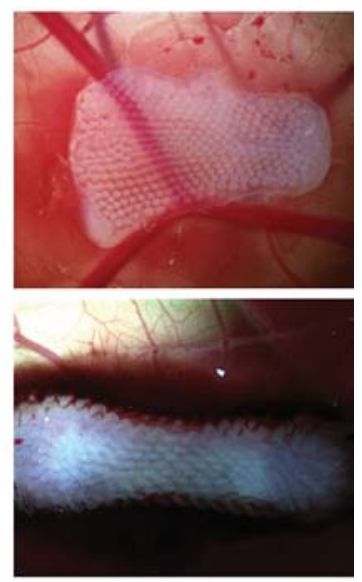

(1 $\mu \mathrm{M})$

Fumagillin (FA)
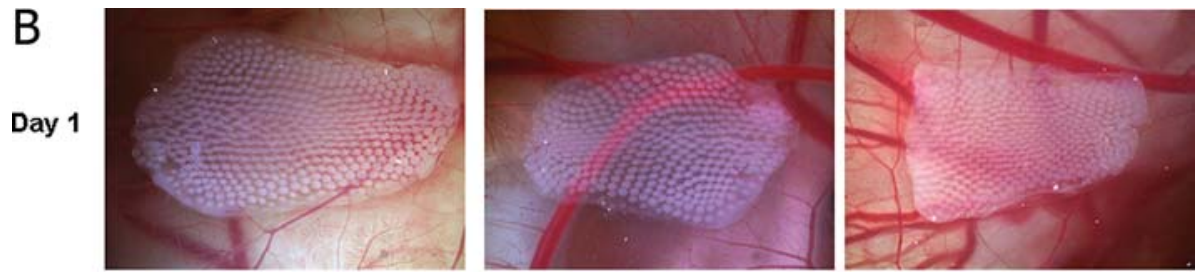

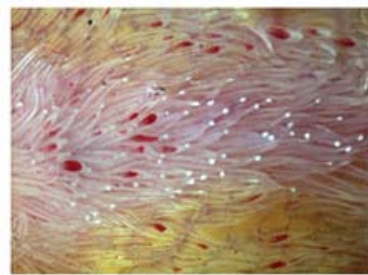

Control (PBS)

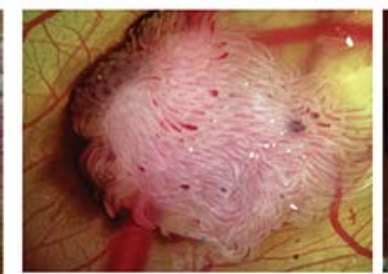

(0.5 $\mu \mathrm{M})$

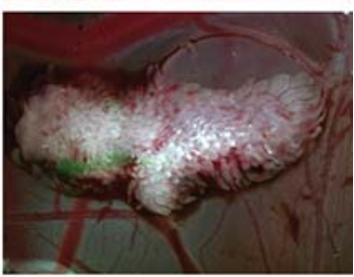

(5 $\mu \mathrm{M})$

Minocycline
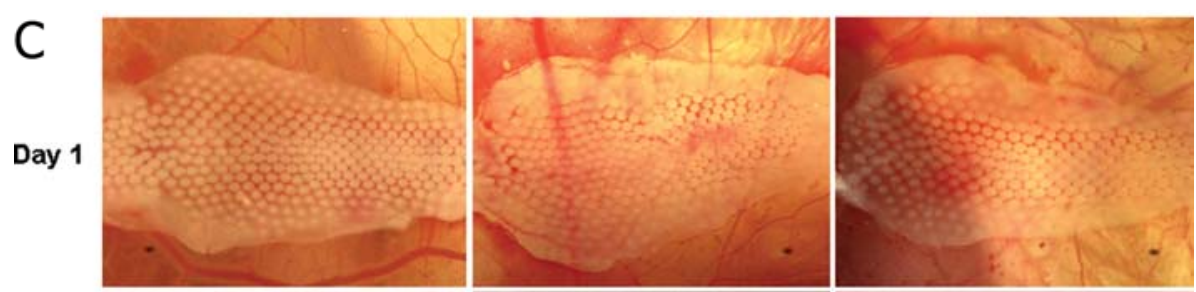

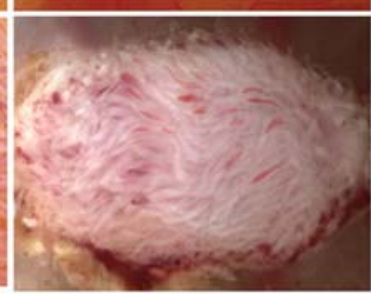

$(2 \mu \mathrm{M})$

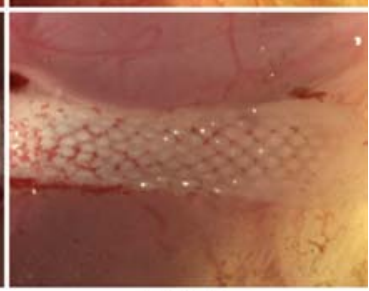

$(8 \mu \mathrm{M})$

Control (PBS)

Zoledronic acid

Figure 2. (A) Inhibition of angiogenesis and FB development with fumagillin which is a known angiogenesis inhibitor. Three groups of E8 embryonic skin tissue explants were cultured in dishes and exposed to the following treatment for $48 \mathrm{~h}$ : 1, PBS alone; $2,0.1 \mu \mathrm{M}$ fumagillin; or $3,1 \mu \mathrm{M}$ fumagillin. After $24 \mathrm{~h}$, there were no visible differences between these groups. However, after 4 days of culture, there was a significant difference between the two fumagillin groups compared to the control group. The results showed fumagillin inhibited angiogenesis and feather development. (B) Inhibition of angiogenesis and FB development with minocycline. Three groups of E8 embryonic skin tissue explants were cultured in dishes and exposed to the following treatment for 48 h: 1, PBS alone; 2, $0.5 \mu \mathrm{M}$ minocycline; or 3, $5 \mu \mathrm{M}$ minocycline. After $24 \mathrm{~h}$ of culture, there were no visible differences between the three groups. However, after 4 days of culture, there was a significant difference between FB exposed to drugs compared to explants that had not been exposed to minocycline. (C) Inhibition of angiogenesis and FB development with zoledronic acid. The nitrogen-containing bisphosphonate zoledronic acid has been shown to inhibit bone loss and recent studies also demonstrate profound anti-angiogenic effects of this drug. We found zoledronic acid markedly inhibits angiogenesis and FB development in the presence of this drug at a concentration of $8 \mu \mathrm{M}$ whereas less effect was observed at $2 \mu \mathrm{M}$. 


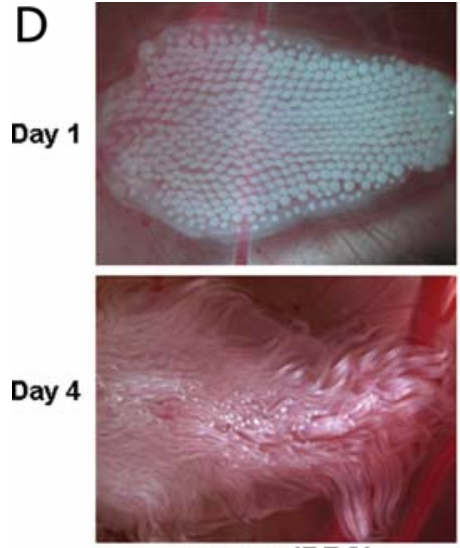

Control (PBS)

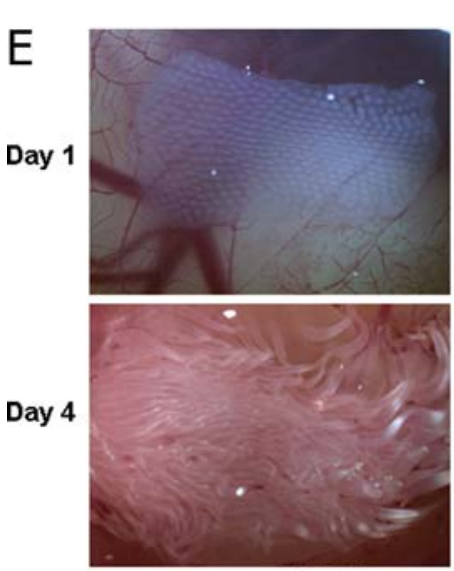

Control (PBS)

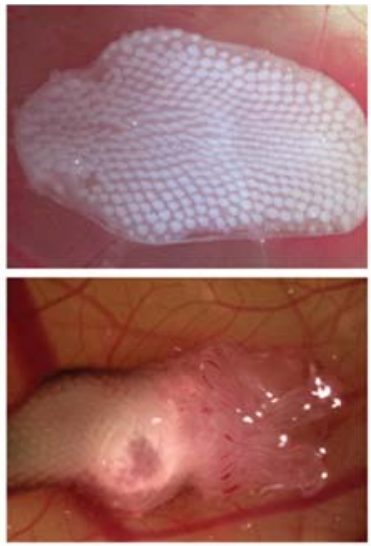

$(0.5 \mu \mathrm{M})$

Doxorubicin
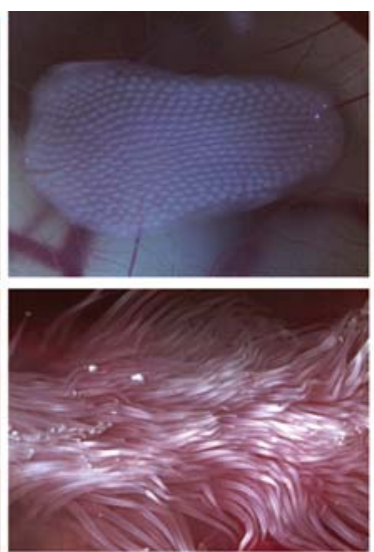

(1 $\mu \mathrm{M})$
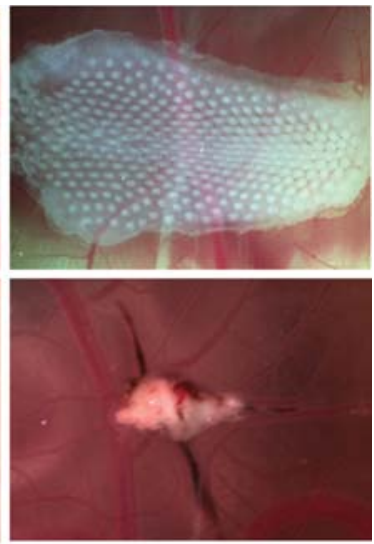

(5 $\mu \mathrm{M})$
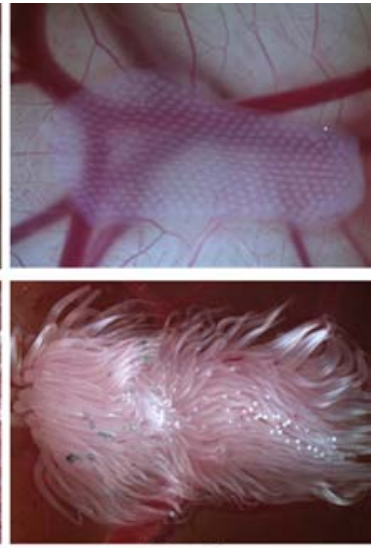

$(10 \mu \mathrm{M})$

Melphalan

Figure 2 (continued). (D) Inhibition of angiogenesis and feather development with doxorubicin which has been shown to possess anti-angiogenic effects. Three groups of E8 embryonic skin tissue explants were cultured in dishes and exposed to the following treatment for 48 h: 1 , PBS alone; $2,0.5 \mu \mathrm{M}$ doxorubicin; or $3,5 \mu \mathrm{M}$ doxorubicin. After 1 day of co-culture, there was no visible difference between these three treatment groups in feather formation. However, by day 4 there was a significant difference between the two doxorubicin-exposed FB groups compared to the PBS alone group. The results showed doxorubicin inhibited angiogenesis and feather development. (E) Melphalan lacks anti-angiogenesis effects. Three groups of E8 embryonic skin tissue explants were cultured in dishes and exposed to the following treatment for $48 \mathrm{~h}$ : 1 , PBS alone; $2,1 \mu \mathrm{M}$ melphalan; or 3 , $10 \mu \mathrm{M}$ melphalan. At $24 \mathrm{~h}$, there was no visible difference between these groups in feather formation. At day 4, there was no difference in the two melphalan-treated FBs compared to the control group.

CA). PCR was performed again using the ThermoScript RT-PCR System (Invitrogen) and a GeneAmp PCR System 9700 (Applied Biosystems, Foster City, CA) for one cycle at $94^{\circ} \mathrm{C}$ for $2 \mathrm{~min}$, followed by 35 cycles at $94^{\circ} \mathrm{C}$ for $30 \mathrm{sec}$, $58^{\circ} \mathrm{C}$ for $30 \mathrm{sec}, 72^{\circ} \mathrm{C}$ for $1 \mathrm{~min}$ and one cycle at $72^{\circ} \mathrm{C}$ for 5 min. Primers: Flk-1 (L) caaccagacggacagtggta, $(\mathrm{R})$ acagact ccctgcttttgct; Tie-2 (L) gcaacttgacttcggtgcta, (R) cacagccgag gagtgtgtaa; GAPDH (L) actgccacccagaagactgt, (R) ccagtagag gcagggatgat.

ELISA for endothelial protein FLK-1. Feather protein lysate $(100 \mu 1)$ from each FB treated with or without fumagillin, minocycline, zoledronic acid or doxorubicin at different concentrations were incubated in flat-bottomed 96 -well microtiter plates overnight at $4^{\circ} \mathrm{C}$. Plates were washed three times with PBS and blocked with PBS containing $0.1 \%$ Tween-20 supplemented with $1 \%$ bovine serum albumin (BSA) at room temperature for $2 \mathrm{~h}$. The plates were then washed three times and incubated with $100 \mu \mathrm{l} /$ well of anti-FLK-1 antibody overnight at $4^{\circ} \mathrm{C}$. Finally, the plates were incubated with alkaline phosphatase-conjugated anti-rabbit $\operatorname{IgG}$ for $1 \mathrm{~h}$ and then washed three times. Bound FLK-1 proteins were detected using BluePhos Microwell Phosphatase substrate (KPL) and analysis using a uQuant (Biotek Industries) plate reader at $450 \mathrm{~nm}$ with KC Junior software. Values represent the mean of triplicate experiments.

\section{Results}

The CAM/FB model is outlined in Fig. 1A. The FB was exposed to agents for 2 days followed by attachment to the CAM for an additional 4 days providing an ideal model through which to quickly and inexpensively evaluate the effects of the drug on blood vessel formation within the FB. If the FB attached to the CAM was exposed to no drugs or compounds that were unable to prevent the development of 
blood vessels, placode and dermal condensation of the FB occurs and the weight of the FB increases as feathers form whereas without attachment to the CAM, FB does not develop blood vessels or feathers (Fig. 1B). To differentiate between the drug's inhibitory effect on preventing blood vessel formation within the FB as opposed to its direct cytotoxic effects on the FB, we exposed the FB to agents such as melphalan for $48 \mathrm{~h}$ which lack anti-angiogenic effects but were directly toxic to the $\mathrm{FB}$ at $30 \mu \mathrm{M}$ whereas drugs showing anti-angiogenic activity including fumagillin (23) and minocycline $(24,25)$ were not toxic to the FB alone (Fig. 1C) at concentrations that produce anti-angiogenic effects (Fig. 2A and B). Furthermore, culture of $\mathrm{FB}$ alone for up to one week with fumagillin showed no cytotoxic effects (data not shown) whereas marked inhibition of FB development occurred in the presence of CAM in a concentration-dependent manner after 4 days of culture (Fig. 2A). Minocycline at a higher concentration (5 $\mu \mathrm{M})$ inhibited FB development and angiogenesis whereas a lower concentration $(0.5 \mu \mathrm{M})$ only slightly reduced FB development compared to tissues without drug exposure (Fig. 2B). Zoledronic acid is a potent nitrogen-containing bisphosphonate that harbors anti-angiogenic activity (26). This drug has been shown to markedly inhibit in vitro proliferation, chemotaxis, and capillarogenesis of bone marrow-derived endothelial cells (27). After 4 days of co-culture with CAM, this agent inhibited feather development at $8 \mu \mathrm{M}$ whereas less effect was observed at $2 \mu \mathrm{M}$ (Fig. 2C). FB alone cultured with zoledronic acid showed no cytotoxic effects. Most recently, anthracyclines including doxorubicin inhibit vascular development in tumor tissues by reducing hypoxia inhibitory factor (HIF)-1 $\alpha$ levels within cells $(28,29)$ whereas melphalan lacks anti-angiogenic activity. Doxorubicin at both 0.5 and $5 \mu \mathrm{M}$ although more so at the higher concentration reduced feather volume and weight and FLK-1 protein expression without showing cytotoxic effects on FB alone (Fig. 2D). In contrast, melphalan which lacks anti-angiogenic activity at concentrations that were not cytotoxic to the FB $(1$ and $10 \mu \mathrm{M})$ did not inhibit feather formation (Fig. 2E). At higher concentrations $(30 \mu \mathrm{M})$, the alkylating agent was cytotoxic to the FB which was also greatly reduced in size or disappeared altogether (Fig. 1C).

Next, we assessed the feather weight and endothelial gene and protein expression. Both feather weight and FLK-1 protein levels were significantly reduced in a concentration-dependent fashion following exposure of the FB to fumagillin, minocycline, zoledronic acid and doxorubicin (Fig. 3A). Both FLK-1 and Tie-2 transcripts and protein levels as assessed with RT-PCR and Western blot analysis, respectively, were significantly reduced in a concentration-dependent manner following treatment of FB with fumagillin, minocycline and zoledronic acid whereas melphalan had no effect on the expression of these genes or proteins (Fig. 3B and C). Compared to the FB attached to CAM without drug, the zoledronic acid-exposed FB attached to CAM lacked organized feather formation and showed a reduction in the number of blood vessels (Fig. 4A). Compared to the FB attached to CAM without drug (Fig. 4B), zoledronic acid inhibited organized feather formation and blood vessel formation in the FB and reduced the number of cells expressing Tie- 2 or FLK-1 (Fig. 4C).
Although the concentration of drugs producing these effects on the FB were much too low to impact the CAM directly when the drug-exposed FB was attached to the CAM, we ruled this potential effect out by attaching doxorubicin $(5 \mu \mathrm{M})$ exposed FB and FB that had not been exposed to drug to the same CAM and showed that only the drug-exposed FB showed inhibition of feather development (data not shown).

\section{Discussion}

Vasculogenesis is defined as the differentiation of precursor cells (angioblasts) into endothelial cells and the de novo formation of a primitive vascular network whereas angiogenesis is defined as the growth of new capillaries from pre-existing blood vessels (30). Angiogenesis involves the development of new blood vessels from pre-existing vasculature $(31,32)$. The pioneering work of Folkman and his colleagues have convincingly established the concept that tumor development is dependent upon neoangiogenesis and have paved the way for the identification of several angiogenic molecules, including the fibroblast growth factor and vascular endothelial growth factor families (33). Several groups have also identified bone marrow-derived endothelial precursor cells and demonstrated that myeloid progenitor, dendritic and mononuclear cells can differentiate into cells of the endothelial lineage. These new endothelial cells circulate and ultimately contribute to blood vessel formation during tumor development and metastasis (34-38). We have recently shown that the secretion of the specific angiogenic proteins pleiotrophin and macrophage colony stimulating factor from malignant plasma cells is capable of stimulating monocytes to become trans-differentiated into endothelial cells that incorporate into tumor blood vessels (9). Because of the critical role that blood vessel formation plays in the development of cancer, therapeutic attempts to block this process have been developed and shown efficacy in some tumors (5-7). To evaluate the effects of potential new agents on angiogenesis, models have been developed to assess the ability of drugs to block endothelial cell development and blood vessel formation. Classic angiogenesis models were based on assessing the formation of capillary-like structures following long-term culture of endothelial cells in the presence of drugs (3). More recently, two-dimensional and three-dimensional angiogenesis assays were developed. In the two-dimensional angiogenesis models, endothelial cells are seeded onto plastic culture dishes that have been coated with either collagen, fibrin, or Matrigel $(12,13,39)$. Threedimensional assays are based on the capacity of activated endothelial cells to invade three-dimensional substrates (40) consisting of collagen gels, plasma clot, purified fibrin, Matrigel or a mixture of these proteins. Unlike our CAM/FB assay, both of these types of models can be used only to measure the number or area of blood vessels in this nonviable material limiting their relevance to living tissues. In addition, these methods only allow evaluation of the effects of drugs on further formation of blood vessels within already vascularized tissues and not on the ability of tissues to attract new blood vessels, so-called angioattraction. Further evaluation of the effect of drugs on angioattraction may lead to the development of new approaches in the treatment of cancer as 
A
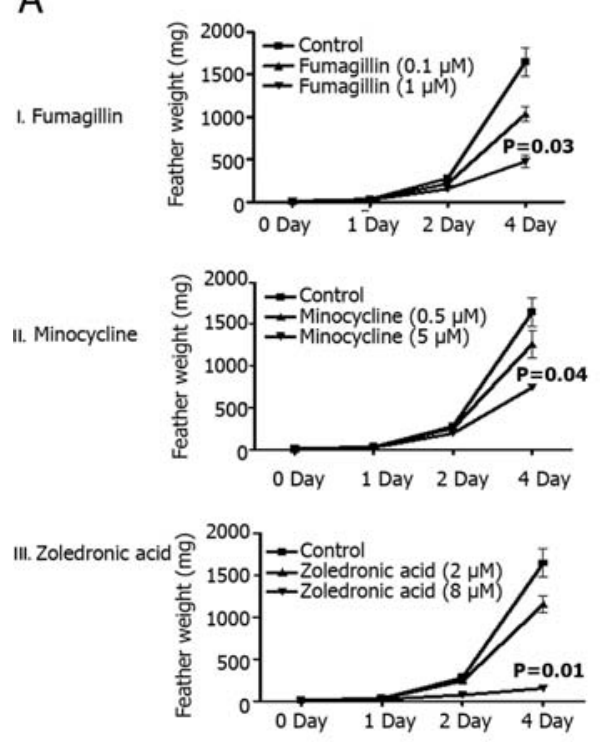

IV. Doxorubicin

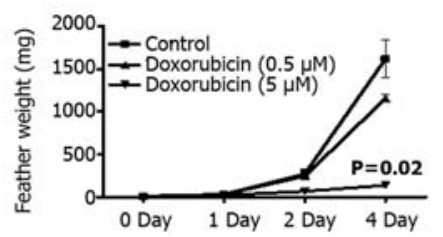

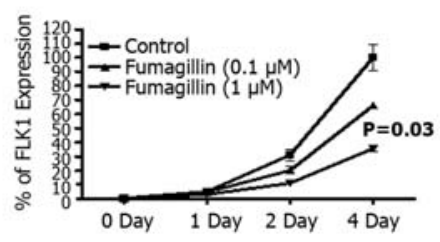
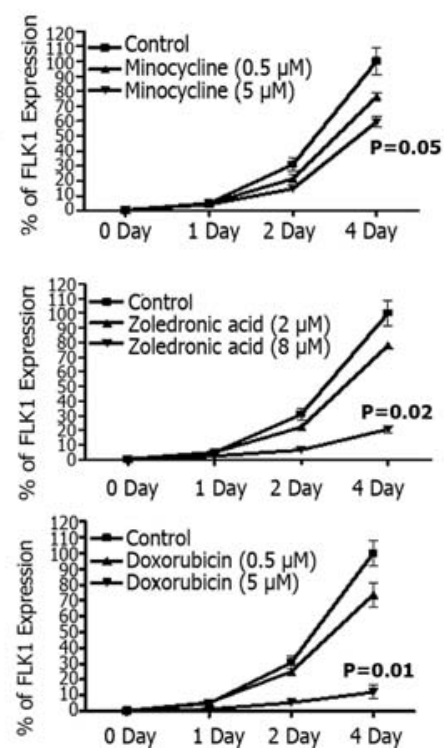

B

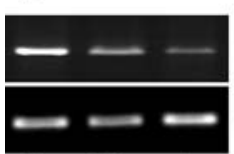

$\begin{array}{lll}0 & 0.1 & 1\end{array}$

FumagillinuM( $\mu \mathrm{M})$

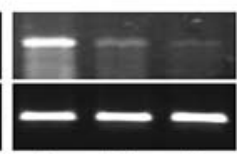

$\begin{array}{lll}0 & 0.5 & 5\end{array}$

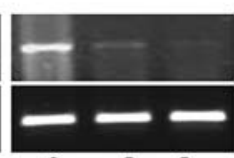

$\begin{array}{lll}0 & 2 & 8\end{array}$

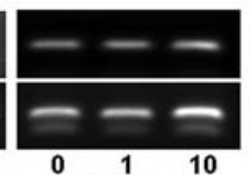

$\begin{array}{lll}0 & 1 & 10\end{array}$

FLK-1

GAPDH

Zoledronic acid $(\mu \mathrm{M})$ Melphalan $(\mu \mathrm{M})$

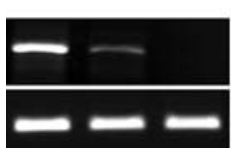

$\begin{array}{lll}0 & 0.1 & 1\end{array}$

Fumagillinum( $(\mathrm{M})$

C

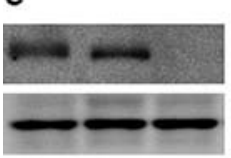

$\begin{array}{lll}0 & 0.1 & 1\end{array}$

$\begin{array}{lll}0 & 0.5 & 5\end{array}$
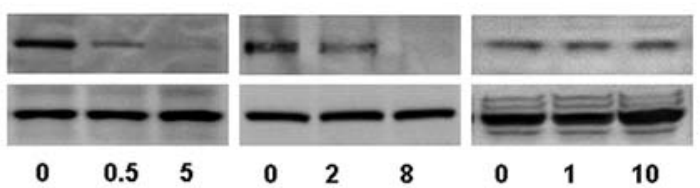

FLK-1

GAPDH

Zoledronic acid( $\mu \mathrm{M})$ Melphalan $(\mu \mathrm{M})$
Tie-2

GAPDH

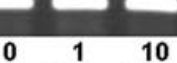

M)

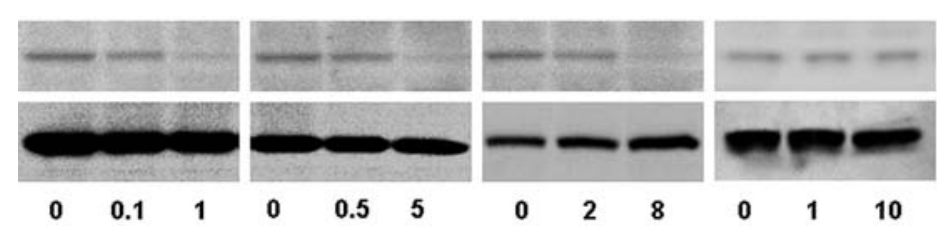

Tie-2

GAPDH

FumagillinuM( $(\mu \mathrm{M})$ Minocycline $(\mu \mathrm{M})$ Zoledronic acid( $\mu \mathrm{M})$ Melphalan( $(\mu \mathrm{M})$

Figure 3. (A) FB was exposed to each agent for 2 days and feather weight and the amount of endothelial protein FLK-1 was determined just prior to and following attachment of the FB to the CAM after 1,2 and 4 days of culture. Control FB without drug treatment was evaluated separately with each drug; 1 , FB treated with fumagillin 0.1 or $1 \mu \mathrm{M} ; 2$, FB treated with minocycline 0.5 or $5 \mu \mathrm{M}$; 3, FB treated with zoledronic acid 2 or $8 \mu \mathrm{M}$; and 4 , FB treated with 0.5 and $5 \mu \mathrm{M}$ doxorubicin. Left, the FB tissue was collected and feather weight assessed using a fine balance. Right, the endothelial marker protein FLK-1 was also quantified using ELISA. (B) Determination of Tie-2 and FLK-1 gene expression with RT-PCR in fumagillin, minocycline, doxorubicin or melphalan-exposed FB attached to CAM for 4 days. (C) Assessment of the amount of Tie-2 and FLK-1 proteins using Western blot analysis in the FB following exposure to fumagillin, minocycline, doxorubicin, or melphalan and after 4 days of attachment to CAM. Statistical analysis, the curves of feather weight and amount of endothelial protein FLK-1 were analyzed in terms of treatment group means and standard error. In order to determine the statistical significance of differences observed in the high concentration treatment groups compared to control groups, a Student's t-test was used for these studies. The minimal level of significance was considered to be $\mathrm{P}<0.05$. 
A

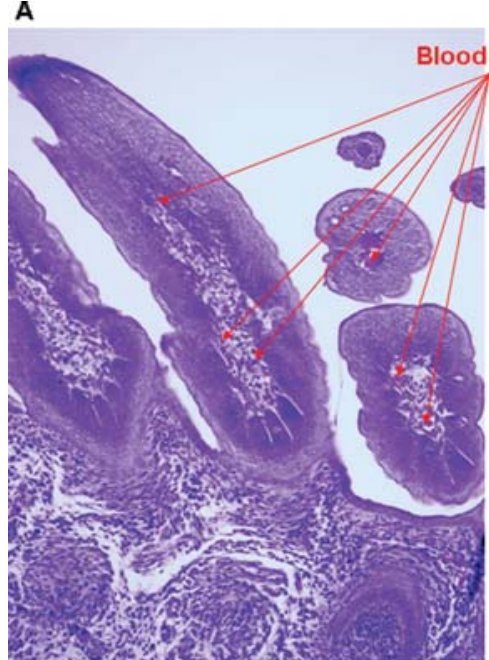

Control

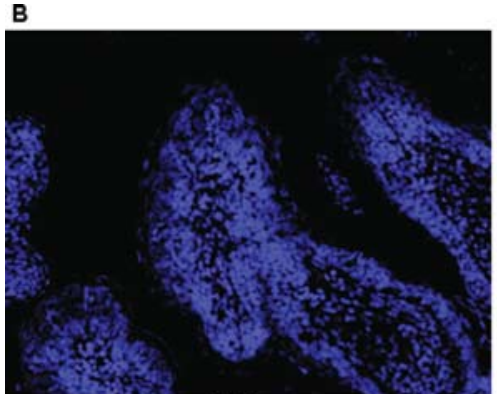

Dapi

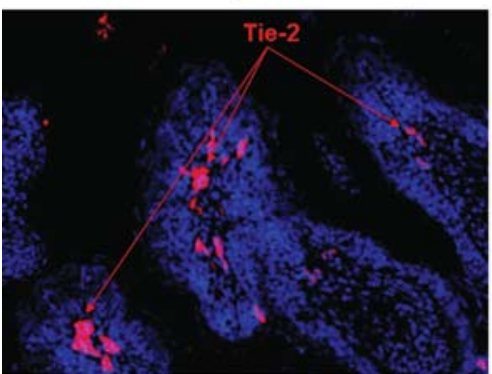

Tie-2

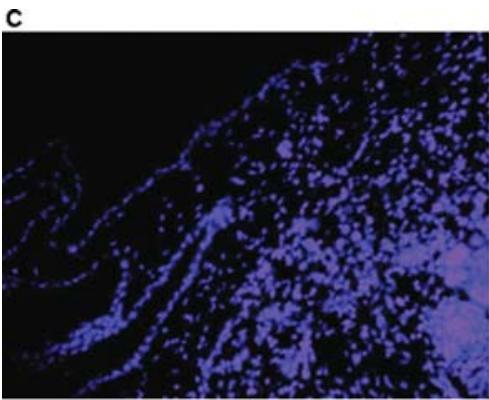

Dapi

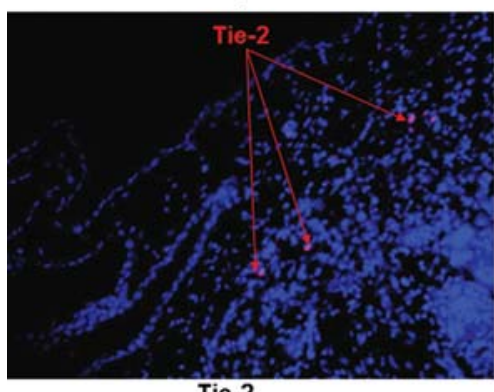

Tie-2

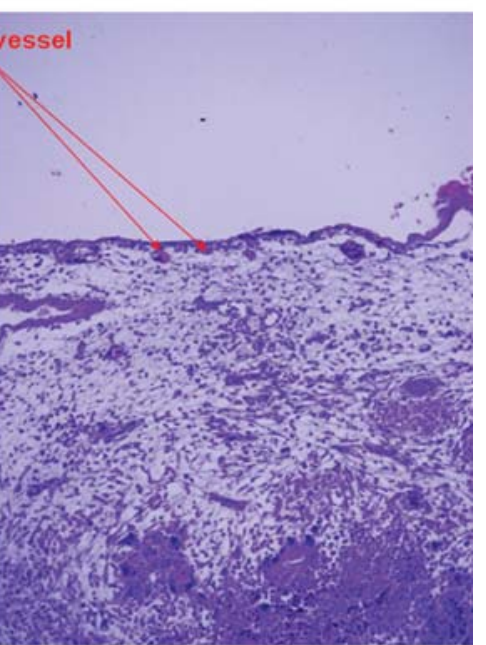

Zoledronic acid $(8 \mu \mathrm{M})$

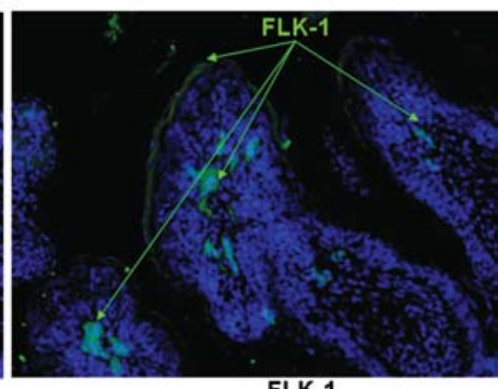

FLK-1

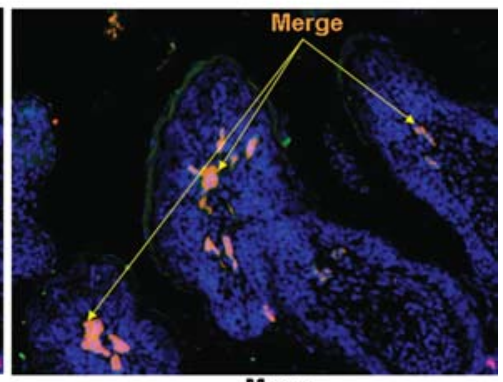

Merge

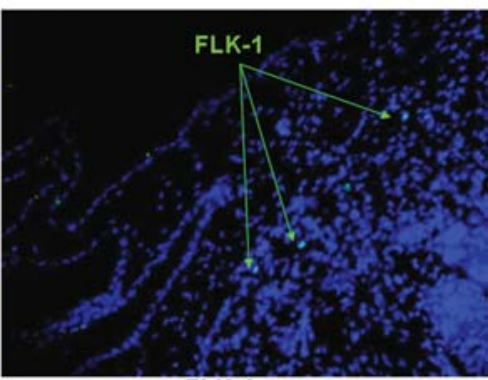

FLK-1

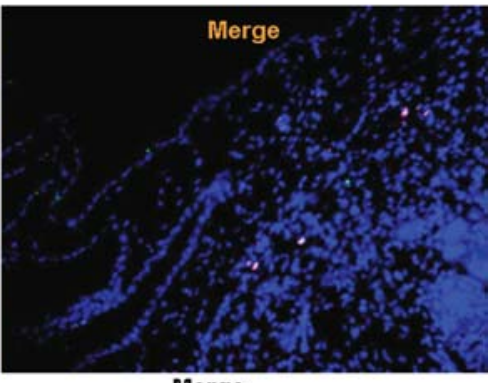

Merge

Figure 4. (A) H\&E staining of the FB attached to CAM for 4 days following exposure of the FB to no drug (control) or zoledronic acid for 2 days. (B) IFA of FB cultured alone for 2 days followed by attachment to CAM for 4 days stained with FLK-1 and Tie-2 antibodies. (C) Similar staining of the FB exposed to zoledronic acid $8 \mu \mathrm{M}$ for 2 days followed by attachment to CAM. 
well as other diseases. Our new CAM/FB model provides an assay to evaluate this phenomenon by using CAM as a way to provide a blood supply to nonvascularized FB tissues that have been exposed to drugs. We have shown that this assay can rapidly and inexpensively assess the effects of a compound in preventing a tissue from attracting and forming its own blood vessels. Thus, the CAM/FB assay opens up a way to evaluate a new avenue, prevention of angioattraction, through which drugs may impact blood vessel formation within tumors and offer the opportunity for new therapeutic approaches for the treatment of cancer.

\section{Acknowledgements}

We are grateful to Christine Pan James for preparing and editing this manuscript. We also thank Geoffrey Gee and Nuhad Barakat for administrative support of this work. This research was supported by the Kramer Foundation (San Francisco, CA), Skirball Foundation (Los Angeles, CA), Annenberg Foundation (Los Angeles, CA), FJC-A Foundation of Philanthropic Funds (New York City, NY), and the Myeloma Research Fund (San Jose, CA).

\section{References}

1. Folkman J: Angiogenesis. Annu Rev Med 57: 1-18, 2006.

2. Andreoli CM and Miller JW: Anti-vascular endothelial growth factor therapy for ocular neovascular disease. Curr Opin Ophthalmol 18: 502-508, 2007.

3. Makale M: Noninvasive imaging of blood vessels. Methods Enzymology: Chapter 8, 444: 175-199, 2008.

4. Hanna E, Quick J and Libutti S: The tumour microenvironment: a novel target for cancer therapy. Oral Dis 15: 8-17, 2009.

5. Sachdev JC and Jahanzeb M: Evolution of bevacizumab-based therapy in the management of breast cancer. Clin Breast Cancer 8: 402-410, 2008.

6. Lakka SS and Rao JS: Antiangiogenic therapy in brain tumors. Expert Rev Neurother 8: 1457-1473, 2008.

7. Hurwitz H, Fehrenbacher L, Novotny W, et al: Bevacizumab plus irinotecan, fluorouracil, and leucovorin for metastatic colorectal cancer. N Engl J Med 350: 2335-2342, 2004.

8. Miller K, Wang M, Gralow J, et al: Paclitaxel plus bevacizumab versus paclitaxel alone for metastatic breast cancer. N Engl J Med 357: 2666-2676, 2007.

9. Chen H, Campbell RA, Chang Y, et al: Tumor-produced pleiotrophin induces transdifferentiation of monocytes into vascular endothelial cells: a novel mechanism of tumor-induced vasculogenesis. Blood 113: 1992-2002, 2009

10. Brown KJ, Maynes SF, Bezos A, Maguire DJ, Ford MD and Parish CR: A novel in vitro assay for human angiogenesis. Lab Invest 75: 539-555, 1996

11. Frater-Schroder M, Risau W, Hallmann R, Gautschi P and Bohlen P: Tumor necrosis factor type alpha, a potent inhibitor of endothelial cell growth in vitro, is angiogenic in vivo. Proc Natl Acad Sci USA 84: 5277-5281, 1987.

12. Dvorak HF, Harvey VS, Estrella P, Brown LF, McDonagh J and Dvorak AM: Fibrin containing gels induce angiogenesis. Implications for tumor stroma generation and wound healing. Lab Invest 57: 673-686, 1987.

13. Montesano R, Orci L and Vassalli P: In vitro rapid organization of endothelial cells into capillary-like networks is promoted by collagen matrices. J Cell Biol 97: 1648-1652, 1983.

14. Grant DS, Tashiro K, Segui-Real B, Yamada Y, Martin GR and Kleinman HK: Two different laminin domains mediate the differentiation of human endothelial cells into capillary-like structures in vitro. Cell 58: 933-943, 1989.

15. Friedlander M, Brooks PC, Shaffer RW and Kincaid CM: Definition of two angiogenic pathways by distinct alpha $v$ integrins. Science 270: 1500-1502, 1995.

16. Chen C, Parangi S, Tolentino MJ and Folkman J: A strategy to discover circulating angiogenesis inhibitors generated by human tumors. Cancer Res 55: 4230-4233, 1995.
17. Miller JW, Adamis AP, Shima DT, et al: Vascular endothelial growth factor/vascular permeability factor is temporally and spatially correlated with ocular angiogenesis in a primate model. Am J Pathol 145: 574-584, 1994.

18. Brooks PC, Strömblad S, Klemke R, Visscher D, Sarkar FH and Cheresh DA: Anti-integrin alpha v beta 3 blocks human breast cancer growth and angiogenesis in human skin. J Clin Invest 96: 1815-1822, 1995.

19. Passaniti A, Taylor RM, Pili R, et al: A simple, quantitative method for assessing angiogenesis and antiangiogenic agents using reconstituted basement membrane, heparin, and fibroblast growth factor. Lab Invest 67: 519-528, 1992.

20. Ribatti D, Vacca A, Roncali L and Dammacco F: The chick embryo chorioallantoic membrane as a model for in vivo research on anti-angiogenesis. Curr Pharm Biotechnol 1: 73-82, 2000.

21. Brooks PC, Clark RA and Cheresh DA: Requirement of vascular integrin alpha v beta 3 for angiogenesis. Science 264: 569-571, 1994.

22. Nguyen M, Shing Y and Folkman J: Quantitation of angiogenesis and antiangiogenesis in the chick embryo chorioallantoic membrane. Microvasc Res 47: 31-40, 1994.

23. Ingber D, Fujita T, Kishimoto S, Sudo K, Kanamaru T, Brem H and Folkman J: Synthetic analogues of fumagillin that inhibit angiogenesis and suppress tumour growth. Nature 348: 555-557, 1990.

24. Passaniti A: Extracellular matrix-cell interactions: Matrigel and complex cellular pattern formation. Lab Invest 67: 804-808, 1992.

25. Yao JS, Chen Y, Zhai W, Xu K, Young WL and Yang GY: Minocycline exerts multiple inhibitory effects on vascular endothelial growth factor-induced smooth muscle cell migration: the role of ERK1/2, PI3K, and matrix metalloproteinases. Circ Res 95: 364-371, 2004.

26. Soltau J, Zirrgiebel U, Esser N, et al: Antitumoral and antiangiogenic efficacy of bisphosphonates in vitro and in a murine RENCA model. Anticancer Res 28: 933-941, 2008.

27. Scavelli C, Di Pietro G, Cirulli T, et al: Zoledronic acid affects over-angiogenic phenotype of endothelial cells in patients with multiple myeloma. Mol Cancer Ther 6: 3256-3262, 2007.

28. Lee K, Qian DZ, Rey S, Wei H and Liu JO: Anthracycline chemotherapy inhibits HIF-1 transcriptional activity and tumorinduced mobilization of circulating angiogenic cells. Proc Natl Acad Sci USA 106: 2353-2358, 2009

29. Zhang J, Sattler M, Tonon G, et al: Targeting angiogenesis via a c-Myc/hypoxia-inducible factor- $1 \alpha$-dependent pathway in multiple myeloma. Cancer Res 69: 5082-5090, 2009.

30. Risau W and Flamme I: Vasculogenesis. Annu Rev Cell Dev Biol 11: 73-91, 1995

31. Carmeliet P: Mechanisms of angiogenesis and arteriogenesis. Nat Med 6: 389-395, 2000.

32. Risau W: Mechanisms of angiogenesis. Nature 386: 671-674, 1997.

33. Folkman J and Shing Y: Angiogenesis. J Biol Chem 267: 10931-10934, 1992.

34. Shi Q, Rafii S, Wu MH, et al: Evidence for circulating bone marrow-derived endothelial cells. Blood 92: 362-367, 1998.

35. Gao D, Nolan DJ, Mellick AS, Bambino K, McDonnell K and Mittal V: Endothelial progenitor cells control the angiogenic switch in mouse lung metastasis. Science 319: 195-198, 2008.

36. Rehman J, Li J, Orschell CM and March KL: Peripheral blood 'endothelial progenitor cells' are derived from monocyte/macrophages and secrete angiogenic growth factors. Circulation 107: 1164-1169, 2003.

37. Bailey AS, Willenbring H, Jiang S, et al: Myeloid lineage progenitors give rise to vascular endothelium. Proc Natl Acad Sci USA 103: 13156-13161, 2006.

38. Conejo-Garcia JR, Benencia F, Courreges MC, et al: Tumorinfiltrating dendritic cell precursors recruited by a beta-defensin contribute to vasculogenesis under the influence of VEGF-A. Nat Med 10: 950-958, 2004.

39. Vernon RB and Sage EH: Between molecules and morphology. Extracellular matrix and creation of vascular form. Am J Pathol 147: 873-883, 1995.

40. Vernon RB and Sage EH: A novel, quantitative model for study of endothelial cell migration and sprout formation within threedimensional collagen matrices. Microvasc Res 57: 118-133, 1999. 